\title{
Emotion classification from EEG signals using wearable sensors: pilot test
}

\section{Clasificación de las emociones a partir de las señales de EEG con sensores portátiles: prueba piloto}

\author{
JARILLO-SILVA, Alejandro†*', GOMEZ-PEREZ, Víctor A.', ESCOTTO-CÓRDOVA, Eduardo A.' \\ and DOMÍNGUEZ-RAMÍREZ, Omar A."”
}

\author{
Universidad de la Sierra Sur, Instituto de Informática, Centro de Tecnologías de la Información, Laboratorio de Interacción \\ Humano Computadora \\ "Universidad Autónoma del Estado de Hidalgo, Research Center on Information and Systems Technology, Electronics and \\ Control Group \\ "Universidad Nacional Autónoma de México, Facultad de Estudios Superiores Zaragoza, Carrera de Psicología, Av. \\ Guelatao No. 66, Col. Ejército de Oriente, alcaldía de Iztapalapa, CDMX
}

ID $1^{\text {st }}$ Author: Alejandro, Jarillo-Silva / ORC ID: 0000-0002-9776-6533, CVU CONACYT ID: 241966

ID $1^{\text {st }}$ Coauthor: Víctor A., Gómez-Pérez / ORC ID: 0000-0002-7758-6690, CVU CONACYT ID: 222462

ID $2^{\text {nd }}$ Coauthor: Eduardo A. Escotto-Córdova / ORC ID: 0000-0002-1104-8195, CVU CONACYT ID: 168236

ID $3^{\text {rd }}$ Coauthor: Omar A., Domínguez Ramírez / ORC ID: 0000-0002-9663-8089, CVU CONACYT ID: 202942

DOI: $10.35429 /$ EJB.2020.12.7.1.9

Received January 19, 2020; Accepted June 29, 2020

\section{Abstract}

The objective of this work is to present a procedure for the classification of basic emotions based on the analysis of EEG signals (electroencephalogram). For this case, 25 subjects were stimulated, of whom 17 were men and 9 women between 20 and 35 years of age. The stimulus to induce positive, negative and neutral emotions with a certain level of excitation (activation) was a set of video clips previously evaluated. The processed and analyzed signals belong to the gamma and beta frequency bands of the F3, F4, P7, P8, T7, T8, O1 and $\mathrm{O} 2$ electrodes. The characteristic variables with the best result are the entropy of each band of each electrode. The cross validation algorithms are applied and later the main component analysis algorithm. Finally, four classifier algorithms are used: classifier trees, Support- Vector-Machine (SVM), Linear-Discriminant-Analysis (LDA) and k-NearestNeighbors $(\mathrm{KNN})$. The results confirm that by carrying out the proposed procedure, the EEG signals contain enough information to allow the recognition of basic emotions.

EEG signal analysis (Electroencephalogram), Machine learning, Recognition of Emotions

\section{Resumen}

El objetivo de este trabajo es presentar un procedimiento para la clasificación de emociones básicas basado en el análisis de señales de EEG (electroencefalograma). Para este caso, se estimularon 25 sujetos, de los cuales 17 fueron hombres y 9 mujeres entre 20 y 35 años de edad. El estímulo para inducir emociones positivas, negativas y neutras con un cierto nivel de excitación (activación) fue un conjunto de videoclips evaluados previamente. Las señales procesadas y analizadas pertenecen a las bandas de frecuencia gamma y beta de los electrodos F3, F4, P7, P8, $\mathrm{T} 7, \mathrm{~T} 8, \mathrm{O} 1$ y O2. Las variables características con mejor resultado son la entropía de cada banda en cada electrodo. Se aplican los algoritmos de validación cruzada y posteriormente el algoritmo de análisis de principales componentes. Finalmente, se emplean cuatro algoritmos clasificadores: árboles clasificadores, SupportVectorMachine (SVM), Linear-Discriminant- Analysis (LDA) y k-Nearest-Neighbours (KNN). Los resultados confirman que llevando acabo el procedimiento planteado, las señales de EEG contienen suficiente información para permitir el reconocimiento de las emociones básicas.

Análisis de señales EEG (Electroencefalograma), Aprendizaje automático, Reconocimiento de emociones

Citation: JARILLO-SILVA, Alejandro, GOMEZ-PEREZ, Víctor A., ESCOTTO-CÓRDOVA, Eduardo A. and DOMÍNGUEZ-RAMÍREZ, Omar A. Emotion classification from EEG signals using wearable sensors: pilot test. ECORFAN Journal-Bolivia. 2020. 7-12: 1-9.

\footnotetext{
* Correspondence to Author (E-mail: ajarillo0205@ gmail.com)

$\dagger$ Researcher contributing first author.
} 


\section{Introduction}

In recent years, the volume of studies and publications on the recognition of emotions has increased. Thanks to technological advancement, techniques and models have been diversified in the generation of systems for the detection of emotions. These systems can be easily differentiated between them; for example, the use of various stimuli, the characteristics for the classification, the size of the time window, the classifiers, the number of participants and the models of emotions. Most research related to emotion detection is mainly based on the biosignal treaty. These can be obtained from different devices, such as; an electroencephalograph (ECG), an electroencephalogram (EEG), galvanic-sensorresponse (GSR), thermal imaging cameras, etc (Koelstra et al., 2011) and in (Li \& Lu, 2009). Thanks to access to technology in recent years the amount of research has increased significantly, where they make use of an EEG (electroencephalograph), however, the field of research is still very wide.

The effectiveness and efficiency of algorithms for the biosignals treaty still show limitations. According to Herger (2013) these include response time, precision, number of electrodes, number of recognized emotions, and the generation of a robust control group database.

The expression of emotions, not only facially, but bodily, mainly involves the bioelectrical activity of various brain structures: the cingulum, the nucleus accumbens, the insula, the orbitofrontal and medial zone, the hypothalamus, the pituitary, the hippocampus, and the amygdala among the most prominent. Unfortunately, with electrodes placed on the scalp that record brain signals (EEG), it is not possible to distinguish or record the relative participation of each one, nor its dynamic participation in each of the possible emotions. However, the signals that are recorded and amplified with an EEG can be correlated with the induction of emotions, especially if a general classification of emotions is used, that is, positive or negative. During the presence of emotions, changes in the electrical signals of the brain are manifested, which are related to the voltage levels (result of ionic current flows within the neurons in the brain) and frequency.
The electrical activity of the brain is classified according to the high and low frequency rhythms called frequency bands. These bands have been defined through studies of psychological and physiological phenomena associated with brain activity.

According to Megías (2011) the study of emotions under experimental conditions has become a fundamental tool to understand the psychological and neurobiological processes involved in their development. Over the last decades, various strategies have been successfully used to induce emotions in experimental conditions (e.g., images, music, videos, self-induction, etc.). Currently the use of scenes from movies (vide films) to induce emotions is one of the most widely used techniques, since it has important advantages such as greater ecological validity since they are stimuli more similar to those perceived in real life. In addition, they are dynamic and different perceptual channels intervene (visual and auditory), allow the standardization and reproduction of the procedure, and allow the induction of specific basic emotions.

In this work, we present a procedure that controls the stimulation of emotions from an intra-subject experimental design, the processing of EEG signals and the evaluation of classification algorithms.

The rest of this document is organized as follows. In Section II a review of the literature is made, describing the models of emotion, stimuli, characteristics, time window and classifier. Section III presents the methodology used in this research. Section IV presents the results obtained. Section V presents a discussion of the results obtained. Finally, Section VI describes the conclusions reached in this work.

\section{Literature review}

The factors that have determined different results in different investigations using an EEG are: emotion model, stimulus, characteristic, time window and the classifier. These factors are described below. 


\section{Models of emotion}

Emotions according to the literature can be classified based on two models: categorical or discrete and dimensional (Bradley \& Lang, 1994) and in (Gray, 2011). The dimensional model proposes that emotional states can be accurately represented by a small number of underlying affective dimensions. That is, it represents the continuous emotional state as a vector in a multidimensional space. Most dimensional models incorporate valence and activation. Valence refers to the degree of "liking" associated with an emotion. It ranges from unpleasant (eg, sad, stressed) to pleasant (eg, happy, exalted). Whereas, activation refers to the force of the emotion experienced. This activation occurs along a continuum and can range from inactive (eg, uninteresting, boring) to active (eg, alert, excited) (Posner, Russell \& Peterson, 2011). This model is used in many investigations for its ease of expressing an emotion in terms of valence and arousal (see Figure 1)

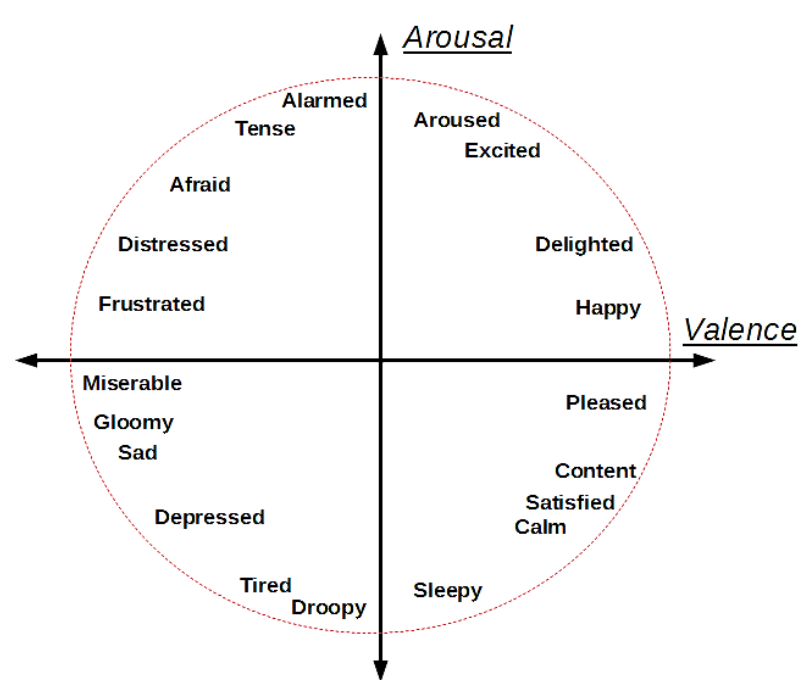

Figure 1 Dimensional model of emotion Source: (Russell, 1980)

Discrete theories of emotion propose the existence of small amounts of separate emotions. These are characterized by coordinated response patterns in physiology, neural anatomy, and morphological expressions. According to Herger (2013) six basic emotions frequently used in research papers include happiness, sadness, anger, disgust, fear, and surprise

\section{Stimuli}

It is defined as the way or way to evoke or induce a certain emotion. Some researchers claim that video clips are the best stimuli, while others find that music or even memories is better. What is clear is that a robust and strong stimulator causes performance with good results, since it guarantees the induction of a certain emotion. Some types of stimulation are: images (NeathTavares \& Itier, 2016), video clips (Daşdemir, Y1ldırım \& Y1ldırım, 2017), music (Daly et al., 2015), memories (Chanel et al., 2015), selfinduction (Iacoviello et al., 2015), games (Chanel et al, 2011), etc. There are generalized databases for the stimulation of emotions, for example, the IAPS (International Affective Picture Systmes), the IADS (International Digitized Sound System) and the GADEP (Geneva Affcetive Picture Database). These databases are the result of the average emotional evaluations of a group of many people.

On the other hand, there are published databases of physiological information collected during the stimulation of a certain emotion. DEAP (Dataset for Emotion Analysis using Physiological) which includes data collected from 32 subjects ( 17 men with an average age of $27.2 \pm 4.4$ years) and SEED (SJTU EEG database) which contains the collection of 62 channels from 15 subjects ( 7 men of $23.7 \pm 2.37$ years), where the subjects were stimulated with 15 videos of 4 minutes each. The induced emotions were positive, neutral and negative.

However, if analyzes of the DEAP and SEED databases are performed, non-conclusive results are obtained, such is the case of the comparison made by Li et al. (2018) where using SEEE an accuracy of $59.06 \%$ and DEAP of $83 \%$ are obtained. It is one of the reasons why this research does not make use of these databases. In Mexico there are various indigenous languages and cultural traditions that influence their categorization, expression and perception. For this reason, the stimulus used is based on video clips, previously validated by the study community. 


\section{Features}

There are a considerable number of variables extracted from EEG signals to be considered representative characteristics of the electrical activity of the brain. In the frequency domain the most widely used is PSD (Power Spectral Density), the power of the EEG signal in its frequency bands using Fourier transform (AlNafjan et al., 2017). In the time domain they are: activity, mobility and complexity, using the Hjorth parameters and the Fractal dimension with the Higuchi method (Yuvaraj \& Murugappan, 2016). In the Wavelet domain: entropy and energy using Wavelet transform (Zhang, et al, 2015). Statistical characteristics: mean, standard deviation, Kurtosis, Skewness, etc (Friedrich et al., 2015).

However, it is interesting to note that nonlinearity in the brain is introduced even at the cellular level, since the dynamic behavior of individual neurons is governed by threshold and saturation phenomena. In this way, on a global level, the brain also presents a really complex and heterogeneous performance, which means that its behavior is far from being considered linear. This research makes use of characteristics based on the study of "log-energy" entropy, which in the described context are justified.

\section{Temporary window}

The appropriate length of the time window largely depends on the type of emotion and physiological signal used. According to Jatupaiboon, Pan-ngum \& Israsena (2013) on average the emotion time is between 0.5 and 4 seconds. When using an inappropriate window, the emotion may be misclassified because different emotions may be present when the periods are too long. Existing literature does not provide an adequate window size to achieve optimal EEG-based emotion recognition. A window size of 1 second is used in this investigation.

\section{Algorithm classifier}

A number of machine learning algorithms have been employed for the classification of emotions, such as SVM (Support Vector Machine), K-NN (K- Nearest Neighbors), LDA (Linear Discriminant Analysis), NM (Naiver Bayes), trees classifiers, ANN (Artificial Neuronal Network), etc.
However, in this research a comparison is developed between K-NN, SVM and classifier trees using the "log-energy" entropy as a characteristic.

\section{Methodology}

The hypothesis that "induced emotions are correlated with electroencephalographic patterns" has been tried with different emotional induction procedures. However, there are different variables, both of the recording of signals (noise, artifacts, etc.) and of the stimuli used in the induction of emotions (images of faces) as characteristics of the participants (race, age, sex, language, culture, etc.) that influence the results. This results in difficulty in inducing a real emotional response using generalized artificial techniques. On the other hand, if a controlled experiment is guaranteed under the same operating conditions, where in addition, the selection of participants meet inclusion criteria, then it is possible to induce an emotional response with the appropriate stimulus.

Based on the above, this section describes the development of two models to predict: one the level of emotion in the dimensions of affective valence (positive, neutral or negative) and the second model the level of activation (high, neutral or low). Figure 2 shows the architecture, which is made up of four main modules: stimulation of the subject, acquisition and processing of the data, extraction of the characteristics and evaluation of the performance of the models. 


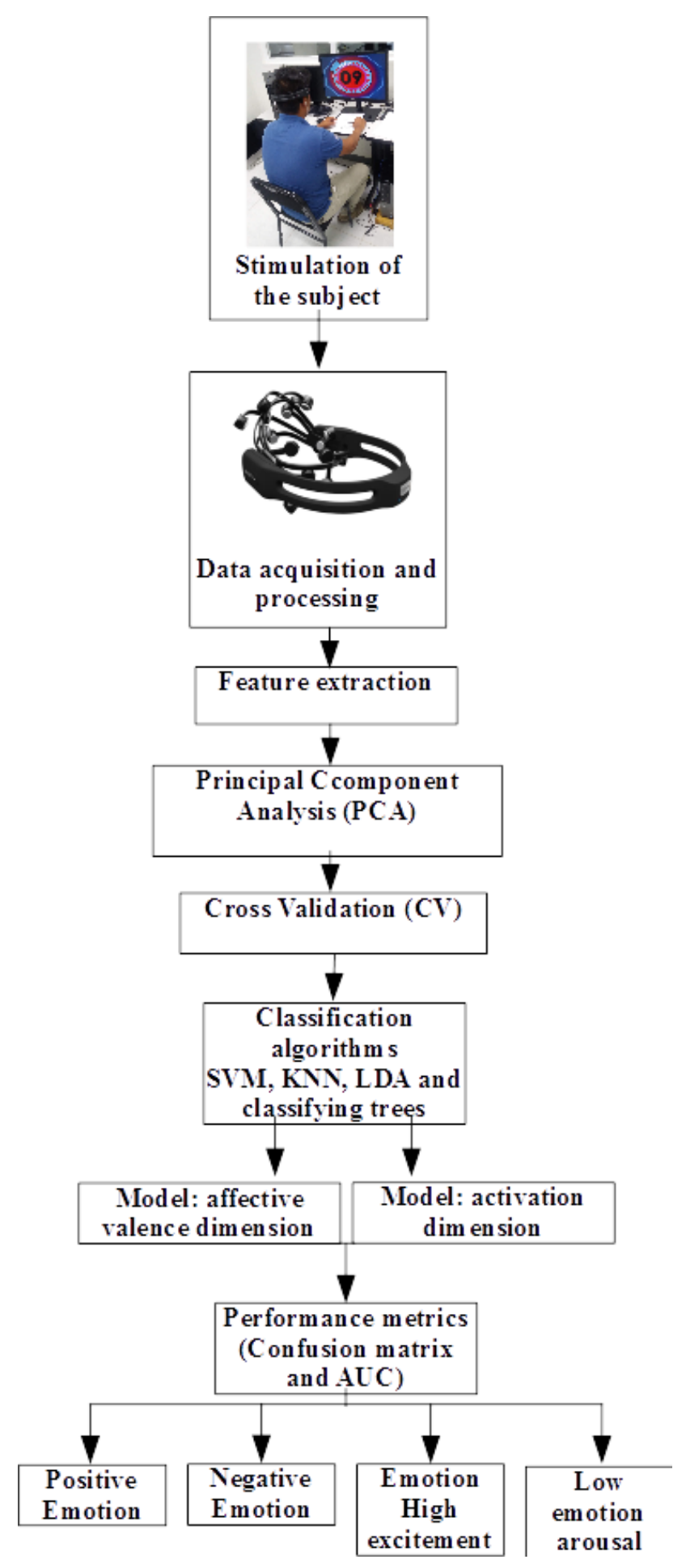

Figure 2 General model architecture

\section{Stimuli}

The experiment involved 25 healthy subjects between 18 and 35 years with an average of 23.7 years, 17 male and 9 female, from the Sierra Sur region of Oaxaca, which were selected for convenience. Exclusion criteria are based on whether the subject is at neurological or psychiatric risk and addictions to alcohol or drugs.
In order to stimulate different emotions in the study subjects, seven validated video clips were used. Each video stimulates a particular emotion with greater intensity. Subjects evaluate each of the videos in terms of valence and excitement using the Self-Assessment Mannequin (SAM) instrument. In these tests, the liker scale is used to quantify arousal and pleasure. They are represented by graphic images that express 9 levels (Morris, 1995).

Figure 3 shows the process for the induction of emotions, which is based on a crosscase study. It begins with the recording of signals from a baseline, in this case the subject remains seated with his eyes open facing a black screen for 35 seconds. Subsequently, the subject is stimulated by a video with a duration of between 3 and 5 minutes. At the end of each video clip you must answer the SAM (Select Assessment for Manufacturing) test, which allows you to know subjectively the assessment of each video in two dimensions (see Figure 4).

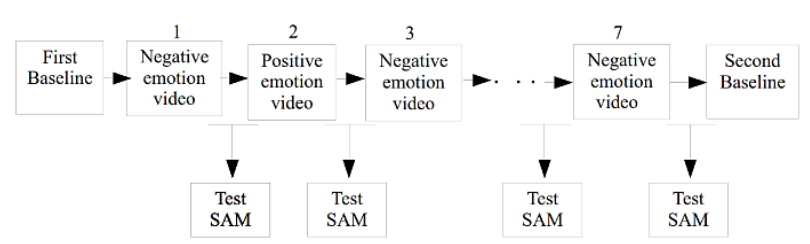

Figure 3 Emotion induction process

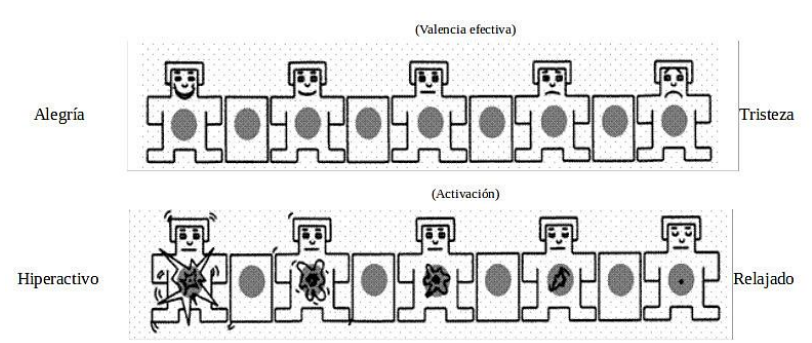

Figure 4 Sam test for two dimensions

After 30 seconds of having finished the first video, the subject is stimulated again with another video, at the end, the subject answers the SAM test corresponding to the immediately previous video, this is repeated until the subject has seen all the videos continuously. At the end of the videos, a second baseline is obtained, which is analyzed and stored in the data processing. The experiment lasts approximately 28.5 minutes. 


\section{Data acquisition and processing}

For this research, the EMOTIV EPOC device is used, which consists of 14 channels (AF3, AF4, FC6, FC5, F3, F4, P7, P8, T7, T8, O1 and O2) located according to the international system 10 -20. Each electrode provides a sampling rate of $128 \mathrm{~Hz}$ and a resolution of 14 bits (see Figure 5. The data is sent to the computer wirelessly using Bluetooth technology. Emotiv Pro software provides raw data for each of the channels, which is stored in a file with the $* \operatorname{csv}$ extension.

Emotiv Epoc device complies with many regulatory requirements like Federal communication commission (FCC) rules part 15 , Radio standard specification (RSS) 210 and low voltage requirement rule: Directive 2006/95/EC. FCC ensures that the device may not cause any harmful interference and the interference received should not cause any undesired operation. RSS-210 is to ensure that the device itself should not cause any interference. (Emotiv, 2016a.)

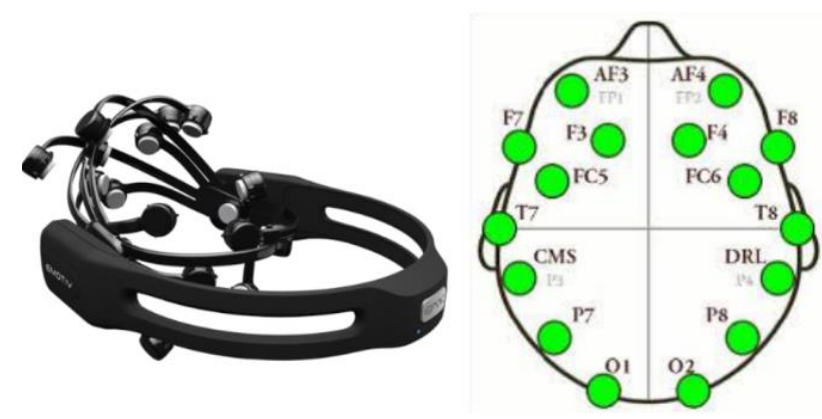

Figure 5 Emotiv device and electrode position system 1020 modified

To manipulate the information extracted from the device, a data grouping method was developed. This method allows organizing and filtering the relevant information, reducing the dimensionality of the data set, in order to search for characteristics that best define the emotional state. This methodology optimized the training and prediction process of the models.

\section{Feature extraction}

Alpha, beta and gamma signals are of great interest in emotion research. However, the presence of noise from artifacts such as eyepieces (below $4 \mathrm{~Hz}$ ), heart sounds (around $1.2 \mathrm{~Hz}$ ) and muscle (above $30 \mathrm{~Hz}$ ) are eliminated by using only the bands of beta and gamma frequency.
On the other hand, nonlinear analysis has proven to be valuable in the evaluation of physiological time series, since hidden information related to the mechanisms has been obtained in a wide variety of clinical settings. However, although there are a large number of nonlinear measurements, entropies based on quantification of the regularity of time series have been widely used in recent years, as they can work as a success even on short and noisy recordings (Pincus, 1991).

\section{Performance evaluation of classifying algorithms}

From the extracted characteristics, the next step is to compare the performance of the classifying algorithms proposed in this investigation. Classifying algorithms are used to provide two models. The valence dimension model has three classes: positive, negative, and neutral. The arousal model consists of three classes; high, low and neutral. In each model, the sensitivity, specificity and percentage of accuracy are obtained. From the Area Under Curve (AUC) it is determined if the model is appropriate to identify emotions.

To avoid overfitting the data when generating each model, it is used k-fold crossvalidation ( $\mathrm{k}$-fold $\mathrm{CV}$ ). This algorithm randomly divides the information into k-1 subsets where $80 \%$ of the data is destined to train and build the model, while $20 \%$ of the remaining data was reserved to evaluate and validate the classifier. The objective of cross-validation is to ensure independence between partitions of training and test data. In this procedure $\mathrm{k}=5$ were used.

On the other hand, the PCA (Principal Component Analysis) algorithm is used. The objective is that from a set of observations of possibly correlated variables they are converted into a set of linearly uncorrelated variables. In this case of a total of 24 variables, 7 linearly uncorrelated variables were found.

\section{Results}

To analyze the results of the SVM, K-NN, LDA and classifier trees algorithms, the confusion matrix was implemented, which contains information about the actual and predicted classification of each algorithm. Table 1 shows the performance of each classifying algorithm in each model.

JARILLO-SILVA, Alejandro, GOMEZ-PEREZ, Víctor A., ESCOTTO-CÓRDOVA, Eduardo A. and DOMÍNGUEZRAMÍREZ, Omar A. Emotion classification from EEG signals using wearable sensors: pilot test. ECORFAN Journal-Bolivia. 2020 


\begin{tabular}{|l|r|r|}
\hline Classifier Algorithms & \multicolumn{1}{c|}{ Valence } & Arousal \\
\hline Classifying trees & $74.3 \%$ & $60.8 \%$ \\
\hline SVM & $83.2 \%$ & $78.8 \%$ \\
\hline KNN & $\mathbf{8 3 . 7 \%}$ & $\mathbf{8 1 . 1 \%}$ \\
\hline LDA & $67.3 \%$ & $47 \%$ \\
\hline
\end{tabular}

Table 1 Accuracy results of each classifier algorithm with the respective model

\section{Performance evaluation of the KNN classifier algorithm}

The classifier algorithm with the best performance in this pilot test is KNN. The KNN algorithm in the affective valence dimension presents a true positive rate of $85 \%$ and a false negative rate of $15 \%$ to identify a negative class. While for the neutral class $15 \%$ of the data identifies it as positive and $14 \%$ as negative (see Table 2). Furthermore, the area under the AUC curve $=0.94$, which is interpreted as the appropriate $\mathrm{KNN}$ algorithm to identify positive, negative and neutral emotions. This means that this classifying algorithm has a better chance of discerning between positive, negative and neutral emotions.

\begin{tabular}{|l|r|r|r|r|r|}
\hline $\begin{array}{l}\text { True } \\
\text { classes }\end{array}$ & \multicolumn{1}{|c|}{-} & \multicolumn{1}{c|}{-} & - & - \\
\hline Negative & $85 \%$ & $12 \%$ & $2 \%$ & $85 \%$ & $15 \%$ \\
\hline Positive & $15 \%$ & $83 \%$ & $2 \%$ & $83 \%$ & $17 \%$ \\
\hline Neutral & $14 \%$ & $15 \%$ & $71 \%$ & $71 \%$ & $29 \%$ \\
\hline $\begin{array}{l}\text { Predicted } \\
\text { classes }\end{array}$ & Negative & Positive & Neutral & $\begin{array}{l}\text { True } \\
\text { positive }\end{array}$ & $\begin{array}{l}\text { False } \\
\text { negative }\end{array}$ \\
\hline
\end{tabular}

Table 2 Confusion matrix of the KNN algorithm for the affective valence dimension

The Table 3 shows the confusion matrix for the arousal dimension. The KNN classifier algorithm presents a prediction accuracy for a high emotion of $82 \%$, while to predict if the emotion is low level it is 79\%. On the other hand, of all the data that correspond to the neutral class, only $18 \%$ incorrectly predict it. The results of the confusion matrix analysis indicate that it has a true positive prediction rate of $82 \%$. While the false positive rate is $13 \%$. The AUC is 0.93 , which indicates that the algorithm is discriminant to define if there is a high arousal level, a very low level or a neutral level.

\begin{tabular}{|l|r|r|r|r|r|}
\hline $\begin{array}{l}\text { True } \\
\text { classes }\end{array}$ & \multicolumn{3}{|c|}{-} & \multicolumn{1}{c|}{-} & - \\
\hline Low & $\mathbf{7 9 \%}$ & $19 \%$ & $2 \%$ & $79 \%$ & $21 \%$ \\
\hline High & $16 \%$ & $\mathbf{8 2 \%}$ & $2 \%$ & $82 \%$ & $18 \%$ \\
\hline Neutral & $8 \%$ & $9 \%$ & $\mathbf{8 2 \%}$ & $72 \%$ & $28 \%$ \\
\hline $\begin{array}{l}\text { Predicted } \\
\text { classes }\end{array}$ & Low & High & Neutral & $\begin{array}{l}\text { True } \\
\text { high }\end{array}$ & $\begin{array}{l}\text { False } \\
\text { low }\end{array}$ \\
\hline
\end{tabular}

Table 3 Confusion matrix of the KNN algorithm for the arousal dimension
Finally, Table IV presents the results of the SAM test evaluation. It is observed that video clips 1, 3, 5 and 7 induce a very low level in the valence dimension, which is interpreted as very negative emotion. Videos 2, 4 and 6 induce a very positive emotion. In the arousal dimension, videos 5 and 7 indicate a high level of excitement while videos 1, 2, 3, 4 and 6 induce a low level of excitation.

\begin{tabular}{|r|l|r|r|}
\hline Videos & Stimulation & Valence & Arousal \\
\hline 1 & Negative & 1.8 & 3.0 \\
\hline 2 & Positive & 6.7 & 2.7 \\
\hline 3 & Negative & 3.2 & 2.8 \\
\hline 4 & Positive & 6.1 & 2.7 \\
\hline 5 & Negative & 1.5 & 4.8 \\
\hline 6 & Positive & 6.7 & 2.1 \\
\hline 7 & Negative & 1.9 & 6.4 \\
\hline
\end{tabular}

Table 4 SAM test results

\section{Discussions}

In this work, we verify the effectiveness of a procedure considering a cross-case study for the induction and recognition of basic emotions. Where entropy is considered as a unique characteristic of the beta frequency bands and range of an EEG. Furthermore, the process allows comparing the performance of classifying algorithms using EEG signals to predict basic emotions.

The results show that the EEG signals contain enough information to distinguish between high, low and neutral in the arousal dimension. While for the valence dimension, distinguish between positive and negative and neutral in affective valence. Furthermore, in particular the KNN classifier algorithm using entropy as its only characteristic is able to distinguish between these classes, although it is worth noting that the SVM model gives results very similar to the KNN.

Subjects play an important role in evaluating classifiers. An attempt was made to select the subjects adequately, so that based on the exclusion criteria, there were no biases in the results. However, although there is an initial baseline, there was no evaluation at the beginning of the experiment that allowed determining the emotional state of the subject. Incorporating information from the subject's emotional state at the start of the experiment probably improves the results of the classifying algorithms. 
The results in Table 4 show that the video clips induce the expected emotion, both in the affective and activation valence dimensions.

This correlates with the results of the KNN classifier model. On the other hand, although tests were made using the 14 electrodes of the Emotiv device, the F3, F4, P7, P8, T7, T8, $\mathrm{O} 1$ and $\mathrm{O} 2$ electrodes were the ones that provide the greatest variability information in emotion detection.

\section{Conclusions}

In this work we have explored the way to distinguish positive and negative emotions, as well as to discern between emotions with a high activation level (excitation) and with a low level.

The use of databases to stimulate emotions does not guarantee favorable results, since it has been seen that much depends on the cultural, social and sometimes even political aspects. According to the results of the pilot test, the use of exclusive videos to stimulate basic emotions in the Oaxaca region of Mexico presents optimistic results.

The use of classifying algorithms in the study of emotions based on EEG information is a way that guarantees encouraging results. Among the algorithms most used for this type of procedure, the KNN provides efficiency and effectiveness in recognizing basic emotions.

However, the cross-validation and PCA algorithms are important to achieve that the classifier algorithm provides favorable results in terms of its sensitivity and specificity.

Finally, it is not necessary to use all the electrodes of the Emotiv device for the study of emotions, with only 8 favorable results were obtained.

In future work, it is intended to use only one pair of electrodes, which will generate sufficient evidence to identify emotions in the affective and arousal valence dimensions.

\section{Acknowledgments}

This research work was supported by PRODEP, IDCA 21306, code UNSIS-CA-13 in the 2018 Strengthening of Academic Bodies call.

\section{References}

Al-Nafjan, A., Hosny, M., Al-Wabil, A., \& AlOhali, Y. (2017). Classification of human emotions from electroencephalogram (EEG) signal using deep neural network. International Journal of Advanced Computer Science and Applications, 8(9), 419-425.

Bradley, M. M., \& Lang, P. J. (1994). Measuring emotion: the self-assessment manikin and the semantic differential. Journal of behavior therapy and experimental psychiatry, 25(1), 4959.

Chanel, G., Kierkels, J. J., Soleymani, M., \& Pun, T. (2009). Short-term emotion assessment in a recall paradigm. International Journal of Human-Computer Studies, 67(8), 607-627.

Chanel, G., Rebetez, C., Bétrancourt, M., \& Pun, T. (2011). Emotion assessment from physiological signals for adaptation of game difficulty. IEEE Transactions on Systems, Man, and Cybernetics-Part A: Systems and Humans, 41(6), 1052-1063.

Daly, I., Williams, D., Hallowell, J., Hwang, F., Kirke, A., Malik, A \& Nasuto, S. J. (2015). Music-induced emotions can be predicted from a combination of brain activity and acoustic features. Brain and cognition, 101, 1-11.

Daşdemir, Y., Yıldırım, E., \& Yıldırım, S. (2017). Emotion Analysis using Different Stimuli with EEG Signals in Emotional Space. Natural and Engineering Sciences, 2(2), 1-10. Emotiv. (2016). Epoc -14 Channel Wireless EEG Headset Description. https://www.emotiv.com/epoc/.

Friedrich, E. V., Sivanathan, A., Lim, T., Suttie, N., Louchart, S., Pillen, S., \& Pineda, J. A. (2015). An effective neurofeedback intervention to improve social interactions in children with autism spectrum disorder. Journal of autism and developmental disorders, 45(12), 4084-4100.

Gray, J. R. (2001). Emotional modulation of cognitive control: Approach-withdrawal states double-dissociate spatial from verbal two-back task performance. Journal of Experimental Psychology: General, 130(3), 436. 
Heger, D., Mutter, R., Herff, C., Putze, F., \& Schultz, T. (2013). Continuous recognition of affective states by functional near infrared spectroscopy signals. In 2013 Humaine Association Conference on Affective Computing and Intelligent Interaction (pp. 832837). IEEE.

Iacoviello, D., Petracca, A., Spezialetti, M., \& Placidi, G. (2015). A real-time classification algorithm for EEG-based BCI driven by selfinduced emotions. Computer methods and programs in biomedicine, 122(3), 293-303.

Jatupaiboon, N., Pan-ngum, S., \& Israsena, P. (2013). Emotion classification using minimal EEG channels and frequency bands. In The 2013 10th International Joint Conference on Computer Science and Software Engineering (JCSSE) (pp. 21-24). IEEE.

Koelstra, S., Muhl, C., Soleymani, M., Lee, J. S., Yazdani, A., Ebrahimi, T., \& Patras, I. (2011). Deap: A database for emotion analysis; using physiological signals. IEEE transactions on affective computing, 3(1), 18-31.

Li, X., Song, D., Zhang, P., Zhang, Y., Hou, Y., \& $\mathrm{Hu}$, B. (2018). Exploring EEG features in cross-subject emotion recognition. Frontiers in neuroscience, 12, 162.

Li, M., \& Lu, B. L. (2009). Emotion classification based on gamma-band EEG. In 2009 Annual International Conference of the IEEE Engineering in medicine and biology society (pp. 1223-1226). IEEE.

Megías, C. F., Mateos, J. C. P., Ribaudi, J. S., \& Fernández-Abascal, E. G. (2011). Validación española de una batería de películas para inducir emociones. Psicothema, 23(4), 778-785.

Morris, J. D. (1995). Observations: SAM: the Self-Assessment Manikin; an efficient crosscultural measurement of emotional response. Journal of advertising research, 35(6), 63-68.

Neath-Tavares, K. N., \& Itier, R. J. (2016). Neural processing of fearful and happy facial expressions during emotion-relevant and emotion-irrelevant tasks: a fixation-to-feature approach. Biological psychology, 119, 122-140.
Pincus, S. M. (1991). Approximate entropy as a measure of system complexity. Proceedings of the National Academy of Sciences, 88(6), 22972301.

Posner, J., Russell, J. A., \& Peterson, B. S. (2005). The circumplex model of affect: An integrative approach to affective neuroscience, cognitive development, and psychopathology. Development and psychopathology, 17(3), 715734.

Yuvaraj, R., \& Murugappan, M. (2016). Hemispheric asymmetry non-linear analysis of EEG during emotional responses from idiopathic Parkinson's disease patients. Cognitive neurodynamics, 10(3), 225-234.

Zhang, C., Tong, L., Zeng, Y., Jiang, J., Bu, H., Yan, B., \& Li, J. (2015). Automatic artifact removal from electroencephalogram data based on a priori artifact information. BioMed research international, 2015. 